\title{
Biosensors based on nucleic acid interaction
}

\author{
M. Minunni \\ Biosensor Laboratory, Department of Chemistry, University of Florence, Via della Lastruccia 3, \\ 50019 Sesto Fiorentino (FI), Italy \\ Tel.: +39055 4573314; Fax: +39055 4573384; E-mail: minunni@unifi.it
}

\begin{abstract}
DNA sensing is an emerging technology based on hybridisation reaction between an immobilised DNA probe and a molecular target, consisting of a probe complementary sequence in solution. Many application have been developed in the field of environmental, food and clinical analysis.

Surface plasmon resonance and piezoelectric sensing are reported as transduction principles for DNA-based devices. These techniques are able to monitor in real-time and without the use of any label the hybridisation reaction between nucleic acids. Particular attention is given to Genetically Modified Organism detection.
\end{abstract}

Keywords: Biosensor, DNA sensing, nucleic acids, hybridisation reaction

\section{Introduction}

The term affinity biosensor refers to a device incorporating immobilised biological receptor molecules that can reversibly detect receptor-ligand interactions with a high differential selectivity and in a non-destructive mode. In affinity sensors, a stoichiometric binding event takes place and the associated physicochemical changes are detected by an appropriate transducer [4]. The biorecognition elements that are normally employed in the realisation of an affinity biosensor are antibodies, receptors or nucleic acids.

In recent years, nucleic acids have received increasing interest as bioreceptors for biosensors and biochips $[1,6,15,20,23]$. The biorecognition mechanism involves hybridisation of deoxyribonucleic acid (DNA) or ribonucleic acid (RNA). The complementarity of adenine : thymine (A:T) and cytosine : guanine $(\mathrm{C}: \mathrm{G})$ pairing in DNA forms the basis for the biorecognition specificity in DNA biosensors. These biosensors are often based on the immobilisation of a fragment of DNA with a specific sequence (probe) and on the monitoring and recording the variation of the transducer signal when the complementary fragment (target) in solution interacts with the probe forming a stable complex.

Gene probes are playing an increasingly important role in health care, agriculture and environmental monitoring $[8,21,22]$.

Quartz Crystal Microbalance (QCM) and Surface Plasmon Resonance (SPR) devices have been extensively applied as transducers in biosensing. In particular, in affinity sensing they have been used in interactions based on immunochemical reactions and also in hybridisation reactions between two complementary nucleic acid strands. Immuno- and DNA sensors based on QCM and SPR devices have been applied with success to real problems from environmental to clinical analysis [5,9-14]. In particular, DNA-based sensors have been presented as alternatives to gel electrophoresis and to traditional methods for specific DNA sequences detection, where labeled (using radioisotope like ${ }^{32} \mathrm{P}$ or fluorescent tags) probes are required. Using QCM or SPR sensing, with an appropriate DNA probe immobilized on the sensor surface, it is possible to detect a specific target sequence in solution.

The transduction principles based on QCM and SPR devices are briefly introduced. 


\subsection{Piezoelectric detection (QCM)}

The converse piezoelectric effect, where an electric field applied to a piezoelectric material indices stress in the material, has been exploited for the detection of minute mass changes. The use of piezoelectric acoustic waves devices and the application of the effect to biosensing has been recently reported [7].

The two most widely studied piezoelectric biosensor configurations are the Quartz Crystal Microbalance (QCM) and the Surface Acoustic Wave (SAW) modes. The QCM devices consist of an electronic circuit that drives an AT-cut quartz crystal, sandwiched between two electrodes, as its fundamental resonance frequency that is tracked with a frequency counter. Changes in the resonant frequency are related to changes in the mass of the crystal, thus allowing the QCM to operate as sensitive mass detector (Fig. 1). SAW devices, operating on the Raleight wave principles can also be used for mass detection by monitoring changes in the acoustic wave propagation between the generator and the receiver sets of electrodes separated by piezoelectric material. Although the ability of these devices to measure gaseous samples has received considerable attention, their most interesting feature is their ability to operate in liquids. Continuous liquid phase operation is always preferable for biosensor design.

In Fig. 2 is shown the recorded signal during the time when binding events occur at the sensor surface. The measurement is in solution. Here a nucleic acid probe is immobilised on the gold, by the use of a dedicated chemistry. After a first addition of buffer (1) to equilibrate the surface, the probe is exposed to the target analyte, a complementary DNA sequence (synthetic oligonucleotide), able to bind the probe. The interaction, due to the hybridisation reaction, causes a mass increase on the crystal surface and, then, a decrease in the resonant frequency of the crystal. The crystal is then washed with the buffer solution to remove the unbound analyte, and the resonant frequency is recorded (2). The difference between (2) and (1) is the frequency shift $\Delta f$ (in Hz) caused by the analyte. Plotting $\Delta f$ versus the analyte concentration,

\section{Piezoelectric sensor}

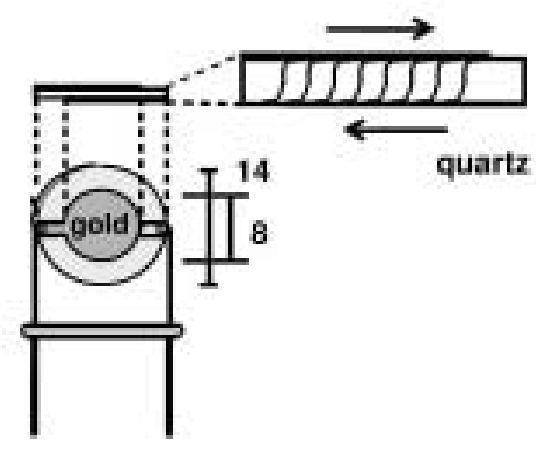

Gravimetric

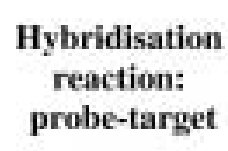

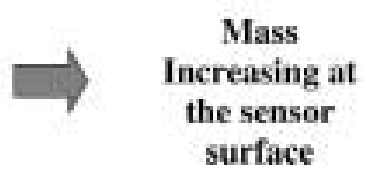

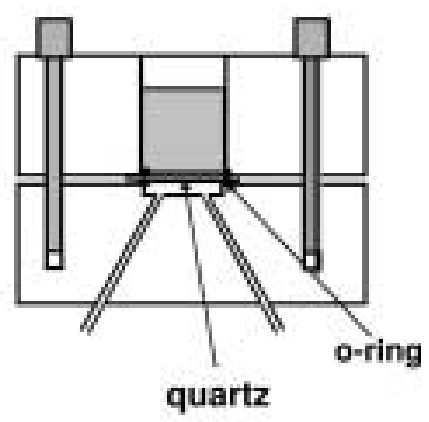

Decrese in

frequency

Fig. 1. Piezoelectric sensing: $10 \mathrm{MZ}$ AT-cut quartz crystal with gold electrodes and measurement cell for analysis in liquid phase. 


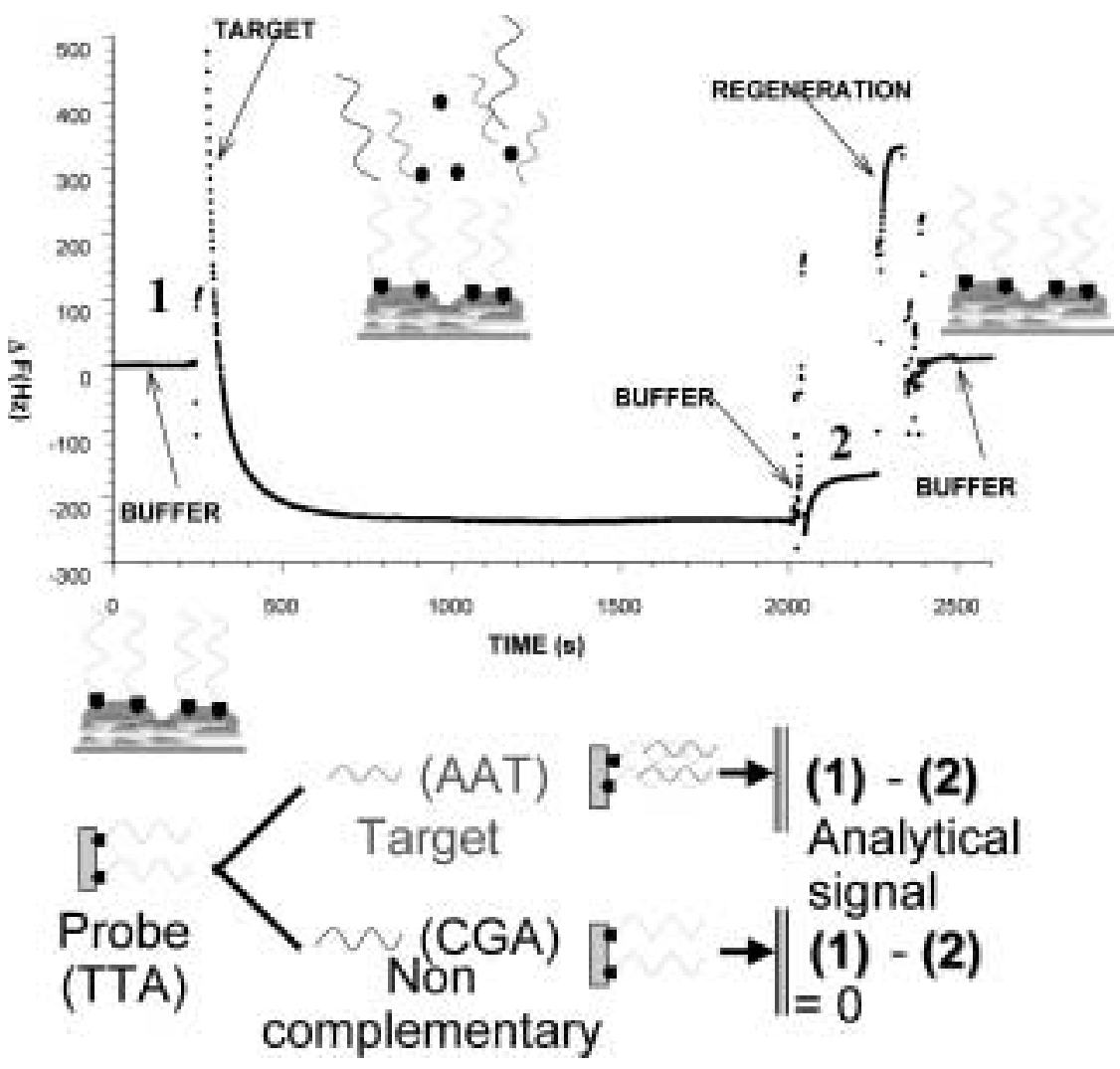

Fig. 2. Piezoelectric sensing: a typical sensor response during DNA hybridisation experiments. The testing sample (target DNA) is injected over the sensing surface with the immobilised probe and the hybridisation interaction occurs. A decrease in frequency can be observed. To remove unbound material the surface is washed with buffer. The surface is regenerated dissociating the affinity DNA bound with an acid agent. The analytical signal is the difference between the value recorded before the sample injection (base line) and the one recorded after the buffer washing.

a calibration curve can be constructed. Form this curve, the unknown concentration of a the target analyte can be estimated.

A problem encountered in the direct detection of mass changes is the effect of non specific adsorption on the sensor response. This can be alleviated to some extent by the use of efficient coatings of the sensing surface aiming to immobilize the receptor responsible of the biomolecular recognition for the biosensing and that, at the mean time, prevent any unspecific adsorption from any other component present in the sample matrix. The comparison between different immobilisation methods, the most used ones, for immobilising biological molecules in QCM sensing has been reported by Storri et al. [16].

\subsection{Surface Plasmon Resonance (SPR)}

Surface Plasmon Resonance (SPR) is a widely used optical method for investigating thin-film phenomena, including binding effects at surfaces. This technique has been adapted for a variety of problems, but has been especially valuable in elucidating biospecific interaction analysis (BIA). The primary impact of SPR in this area is the ability to monitor the binding interactions based on affinity reaction, i.e., antigenantibody binding, ligand-receptor, nucleic acid hybridisation, etc. One is able to conduct studied based on not only binding equilibrium data, but also kinetic of the on-off binding processes. 
The principle of SPR is based on the creation of surface plasmons in a metal layer. One can envision of a surface wave created by an oscillating electric charge on the surface of the metal. This oscillating charge density is called plasmon. For the purpose of this discussion it is sufficient to say that the surface plasmon of the metal layer cannot be excitated by the incident light due to restrictions dealing with the laws for conservation of energy and momentum. The plasmon can be induced, however, by the evanescent wave from total internal reflection using TM polarized light, where TM stands for transverse magnetic mode. The surface plasmon effect is extremely sensitive to the refractive index and thickness of films on opposite surface of the metal layer.

Usually is used Kretschmann configuration for SPR. Light undergoes total internal reflection so that the evanescent wave interacts with a thin metal film on the surface of the prism. Silver and gold have the highest optical constant for this purpose. The incident angle of coherent, TM polarized light is varied. At some particular incidence angle the electric vector of the incident light induces a resonance with the surface plasmons.

If a thin film of differing dielectric is immobilized on the opposite metal surface, the evanescent wave of the surface plasmon couples to that layer.

Chemical changes in that film then modulate the reflected light from the prism. The reflected light is monitored and the resonant condition is indicated by a decreasing in intensity of the reflected light as function of the incident angle. The angle at which the minimum occurs is refereed to as the SPR angle (Fig. 3).

For example, a resonance valley would occur at one angle of incidence for an antibody (Ab) immobilized on the metal surface, whereas the SPR angle would shift when the antibody binds to the antigen $(\mathrm{Ag})$ in a sample. The affinity interaction between $\mathrm{Ab}-\mathrm{Ag}$, leading to the immuno-complex formation can be then monitored in real-time.

The hybridisation interaction between nucleic acids is also an affinity interaction. A synthetic oligonucleotide is immobilised on the sensor surface and the complementary strand is free in solution. Likely

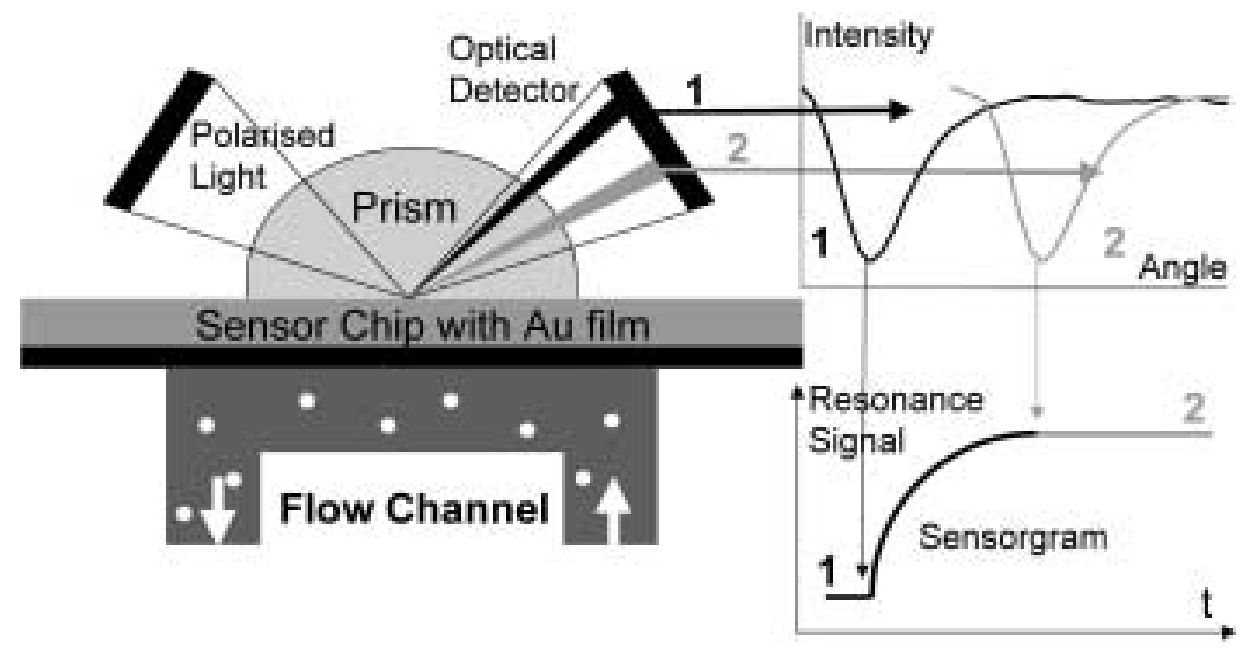

Fig. 3. SPR sensing: In condition of total internal reflection, the evanescent wave generates plasmons and a dip in the intensity of the reflected light is observed. The incident angle at which the minimum is recorded is called resonant angle. The resonant angle influenced by the refractive index in the medium where the interaction occurs is monitored. The graph displacing the variation of the resonant angle vs time is the sensorgram. 
to the $\mathrm{Ab}-\mathrm{Ag}$ complex formation, the hybrid formation between the two nucleic acids strands can be monitored in real-time.

An example of interaction between a probe immobilized on the surface and the complementary target in solution is reported in Fig. 4. Likely as for QCM transduction, the measurement is in solution and allows one to follow the hybridisation reaction as it occurs. After a first addition of buffer (1) to equilibrate the surface, the probe is exposed to the target analyte, a complementary DNA sequence (synthetic oligonucleotide), able to bind the probe. The interaction, due to the hybridisation reaction, causes a change in the refractive index which influence the resonant angle recorded as a shift (2). The difference between (2) and (1) is the resonance shift, expressed in arbitrary Resonance Units (RU). Plotting $\Delta R U$ versus the analyte concentration, a calibration curve can be constructed. Form this curve, the unknown concentration of the target analyte can be estimated.

The most representative instrument based on this technology is the BIACORE ${ }^{\mathrm{TM}}$ (Biacore AB, Uppsala, Sweden).

Both piezoelectric and SPR detection principle allows one to follow the biospecific interaction while they are occurring, in real-time, and without the use of any label. For this reason they represent two very interesting transduction principles for biosensors development.

The application area for those biosensors is wide: from environmental to food, to clinical analysis.

In order to act as biosensor, the bioreceptor has to be immobilized on the sensor surface.

Many immobilisation methods have been developed for immobilising molecule on the metal layer; gold is almost always employed and dedicated chemistries have been applied with success [2,16,17]. The covalent binding of biomolecules should not affect its biological activity. For the reproducibility of

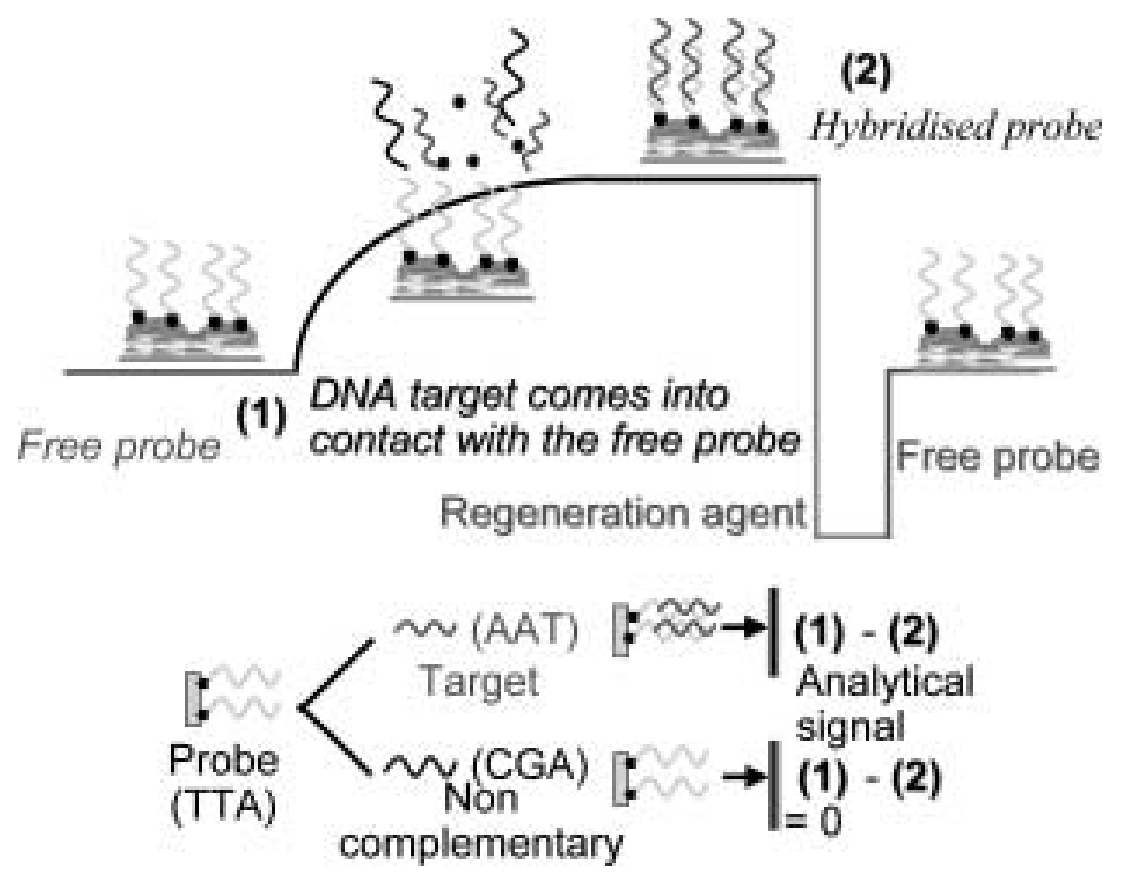

Fig. 4. A sensorgram displaying the affinity interaction between the immobilised probe and the complementary target in solution. While the interaction occurs the increase in the sensor signal can be observed. The system operates in flow. After the washing of the surface the hybridisation signal is taken as the difference between the values recorded before the interaction (base line) and after the washing. 
the analytical signals it is also important that there is no leakage of the ligand from the surface and that there is no unspecific adsorption of compounds eventually present in the sample.

Both systems, QCM and SPR, are prone to errors due to unspecific adsorption since they are only sensitive, respectively, to mass and refractive index changes. If a compound unspecifically binds the surface it will give an analytical signal. For these reasons the coating and the immobilization chemistries are a key step in biosensors' development.

The approach reported below was successfully developed more then 10 years ago at Pharmacia Biosensor AB (Uppsala-Sweden) by Löfås et al. [2]. A dextran layer was successfully employed to minimize the unspecific adsorption problem.

This chemistry has been widely applied for the development of many different biosensors: immuno, receptor, and finally DNA based sensors.

For the development of DNA-based sensors a probe should be immobilised on the sensor surface. The probe can be either immobilised directly, without the use of a linking layer or better employing anchoring via streptavidine protein. In this latter case biotinylated probe are required. In the development of DNAbased sensors biotinylated probes are mostly employed.

\subsection{Immobilisation of oligonucleotides' probes on sensors' surfaces}

The immobilisation chemistry uses, as substrate for probes' immobilisation, a gold surface; in fact, the quartz crystal is sandwiched between two gold electrodes, and the SPR chip consists in a glass coated with gold layer. The chemical modification occurs, in both cases, at the gold level.

When a biosensor is developed a key step is the immobilisation of biomolecule.

It is usually better to immobilise covalently the molecule on the sensor surface, using chemical binding instead of a physical adsorption of the molecule itself. For this reason many different immobilisation chemistries have been developed. In piezoelectric and SPR sensing is very important that any possible unspecific interaction, from the solution with the surface, is prevented. The below reported chemistry has been usefully applied to both these transduction principles since it fulfill the here mentioned requirements.

At the basis of this coupling there is the strong interaction between thiols and gold. For this reason an alkan-thiol is first employed (linking layer). Secondly, a carboxylated dextran hydrogel matrix is created. This was demonstrated to minimize macromolecules denaturation. In addition dextran has a very low unspecific adsorption for biomolecules. The hydrogel is chemically stable in water, in buffers used for biomolecular interaction studies, in weakly alkaline and acidic solutions. The carboxylated dextran, is activated for ligand binding through derivatisation with $n$-hydroxy succinimide. $30-40 \%$ of dextran carboxylic groups are converted into reactive esters of NHS. These NHS-esters reacts with primary aminogroup present on the ligand. Many biological molecules can be bound via their primary aminogroup without loosing their biological activity.

After the ligand binding to the hydrogel, the remaining NHS-esters are deactivated with ethanolamine leading to hydroxyethylamide replacing activated groups.

In DNA sensing this immobilization chemistry has bee used to immobilize as ligand, first streptavidine. Streptavidine is a tetrameric protein showing a very high affinity for biotin with a $K_{\mathrm{A}}$ in the order of $10^{15}$. By anchoring biotinylated probes to streptavidin layers, a stable DNA surface is created for interaction with complementary target DNA (Fig. 5).

This is the chemistry employed in many approaches we have developed. The QCM has been coupled with polymerase chain reaction, i.e., for bacterial toxicity (Aeromonas) determination in environmental 
Gold
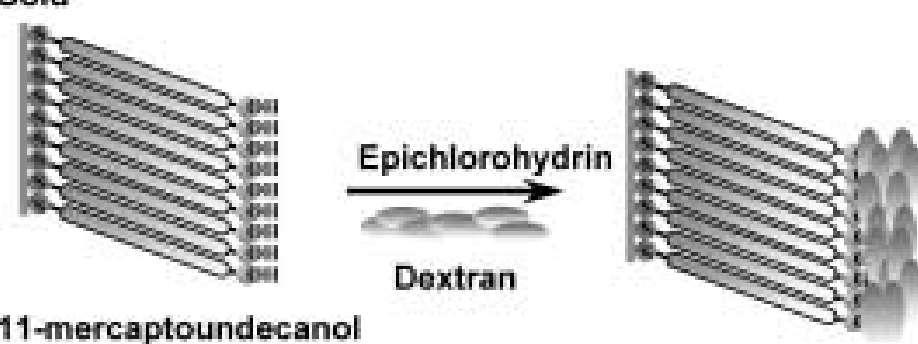

11-mercaptoundecanol

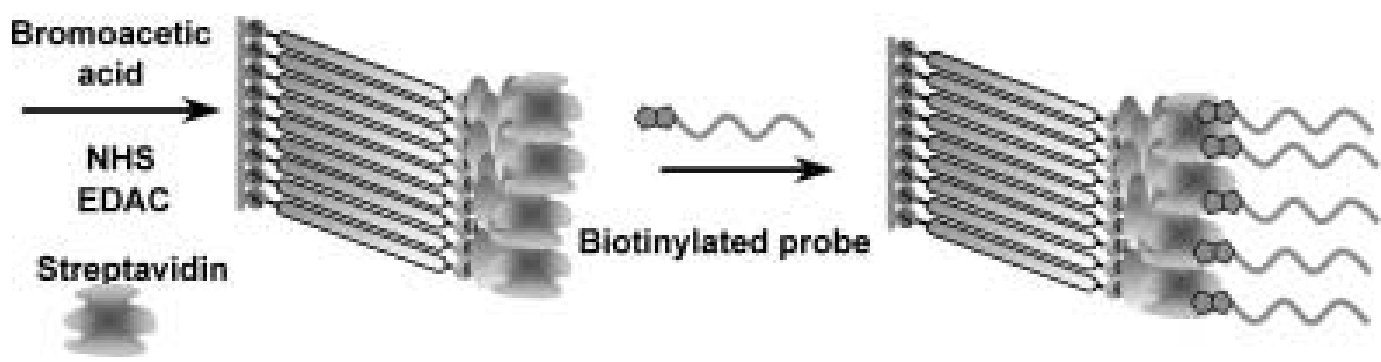

Fig. 5. Immobilisation chemistry (see text).

samples [18], and to detect apolipoprotein E polymorphisms in serum samples with application to clinical diagnostic [19]. In both cases the specific DNA probe were immobilised on the transducer.

Recently we approached the problem of Genetically Modified Organism (GMOs) detection. A new probe, specific for the target molecule, analyte, is immobilised. Below follows the description of the biosensor development for GMOs detection using QCM and SPR transduction principles.

\section{Application of QCM and SPR to Genetically Modified Organism (GMOs) detection}

DNA hybridisation techniques coupled with QCM or SPR devices can be powerful tools also in food analysis because they offer high sensitivity and specificity. In addition, they may offer improved technology for the detection of organisms that are difficult to isolate from food, can be applied to more rapid real-time analysis of food samples, and may be the most applicable technique to quantify viral elements or genetically modified organisms (GMO).

The steady increase of the use of GMOs in the food chain, impose the development of new procedures for the certification of food components origin. In Europe labelling is mandatory for foodstuff containing ingredients derived from genetically modified maize (Bt-maize from Novartis) and soy beans (RR-soy from Monsanto). The control and detection of foodstuff is an important issue and screening methods to identify GMOs presence are strongly requested. The current available GMOs detection methods are expensive and time requiring, thus biosensors could represent an alternative to them $[13,14]$.

The QCM and SPR biosensors have been developed by first immobilising a specific probe ( 25 bases long) using the same immobilisation chemistry for the two transducers (quartz crystals with gold electrodes for the QCM device and glass/gold chip for the SPR device as reported above and shown in Fig. 5).

We have to know first, that genetic modification takes place in a structural gene or in sequences that regulate gene expression. One regulatory sequence found in the majority of GMO plants has focused the interest of research scientists. This sequence is contained in the $35 \mathrm{~S}$ promoter region of Cauliflower 
A gene cassette consists of a promoter (P), coding region and a terminator $(\mathrm{T})$

\begin{tabular}{|c|c|c|}
\hline Prom. & Coding Region & Term. \\
\hline & &
\end{tabular}

Fig. 6. A gene cassette consisting of a promoter, a coding region and a terminator. In most of genetically modified organisms available on the market the promoter sequence is derived from Cauliflower mosaic virus P35S.

Mosaic Virus (CAMV) ribosomal RNA; for this, a probe with a sequence internal to the CAMV sequence is chosen for the biosensors development. In Fig. 6 is reported a gene cassette consisting of a promoter a coding region and a terminator. The promoter corresponds to P35S. The probe and the target sequences are reported below:
Probe:
5'-biotin-GGC CAT CGT TGA AGA TGC CTC TGC C-3'
Target:
$5^{\prime}$-GGC AGA GGC ATC TTC AAC GAT GGC C-3'
Non complementary:
5'-GAT TAG AGT CCC GCA ATT AAT CAT T-3'

A control sequence, non complementary (negative control) to the probe, is normally used to check the specificity of these devices. For an optimised chemistry no signal should be found with the negative control.

Both the biosensors are optimised with 25-mer synthetic oligonucleotides for sensitivity and selectivity determination. The optimised biosensors have been then applied to the analysis of P35 PCR amplified DNA sequences.

Below are reported the two systems employed for GMO detection based on SPR and piezoelectric transduction principles.

\section{Sensors' design}

\subsection{Surface Plasmon Resonance (SPR)}

BIACORE $^{\mathrm{TM}} \mathrm{X}$ instrument (Biacore AB, Uppsala, Sweden) is the instrument based on SPR detection principle, employed for GMO detection. The probe oligonucleotides are immobilised on a dextrancoated chip (CM5 chip, Biacore AB, Uppsala, Sweden) and the experiments could be conducted in flow $(5 \mu \mathrm{l} / \mathrm{min})$ and with a controlled temperature $\left(25^{\circ} \mathrm{C}\right)$.

\subsection{QCM apparatus}

Most crystal employed in piezoelectric sensing use gold electrodes, evaporated on both sides.

$10 \mathrm{MHz}$ AT cut quartz crystals represent a good sensing surface for immobilising DNA probes. The quartz crystal, after the immobilisation steps, can be housed inside a methacrylate cell (Fig. 1) such that only one side of the crystal is in contact with the solution in the cell well.

The frequency variations can be continuously recorded using a quartz crystal analyser. The frequency shifts can be reported as the difference between two stable frequency values $( \pm 1 \mathrm{~Hz})$ before and after the affinity binding.

With these systems many different DNA sources at increasing complexity can be tested for GMO detection, i.e. from plasmidic to plant DNA. Here are reported some examples of tested DNA with our biosensors. 


\subsection{DNA samples}

Plasmidic DNA from pBI121, maize DNA samples from animal feeding and transgenic DNA from Nicotiana glauca plant. Are reported as example of samples containing the target sequence (promoter P35S DNA) detected by QCM and SPR biosensors. The DNA to be tested should be extracted and amplified from the original source.

Many protocols are available, but an optimised one for GMO detection is reported by Pietsh et al. (1997), adopted as European screening method for GMO detection. The amplification of P35S is conducted via polymerase chain reaction (PCR) and results (using the protocol recommended primers) in a fragment of $195 \mathrm{bp}$.

Using separate PCR procedures, with different primers, it is possible to amplify fragment of P35S of different length: 243 or 492 bp.

\subsection{Immobilisation of the oligonucleotide probe}

The immobilisation of the oligonucleotide probes in both systems uses the strong affinity between biotin and streptavidin and refers to previously described immobilisation chemistry.

\subsection{Hybridisation experiments}

\subsubsection{Sensors' calibration}

The recorded signals of the hybridisation reaction are obtained as shown in Figs 2 and 4 both from SPR and QCM hybridisation experiments. The analytical signal in both cases is provided by subtracting the values of the baseline before and after the hybridisation reaction. Calibration curves with the 25 -mer

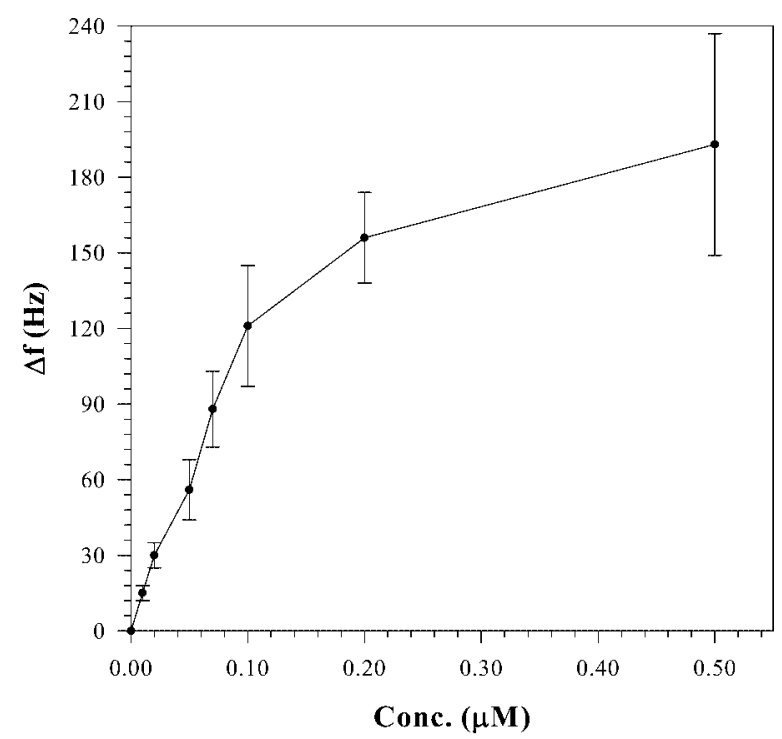

Fig. 7. Piezoelectric sensor: Calibration curve obtained with the 25 -mer complementary oligonucleotides for P35Sprobe. In the right the zoom of the linear region is reported. $100 \mu \mathrm{l}$ of sample are added in the cell and the hybridisation reaction is allowed to proceed for 10'. In all the experiments the single stranded probe was regenerated by 1 min treatment with $1 \mathrm{mM} \mathrm{HCl}$ allowing a successive hybridisation reaction to be monitored. 


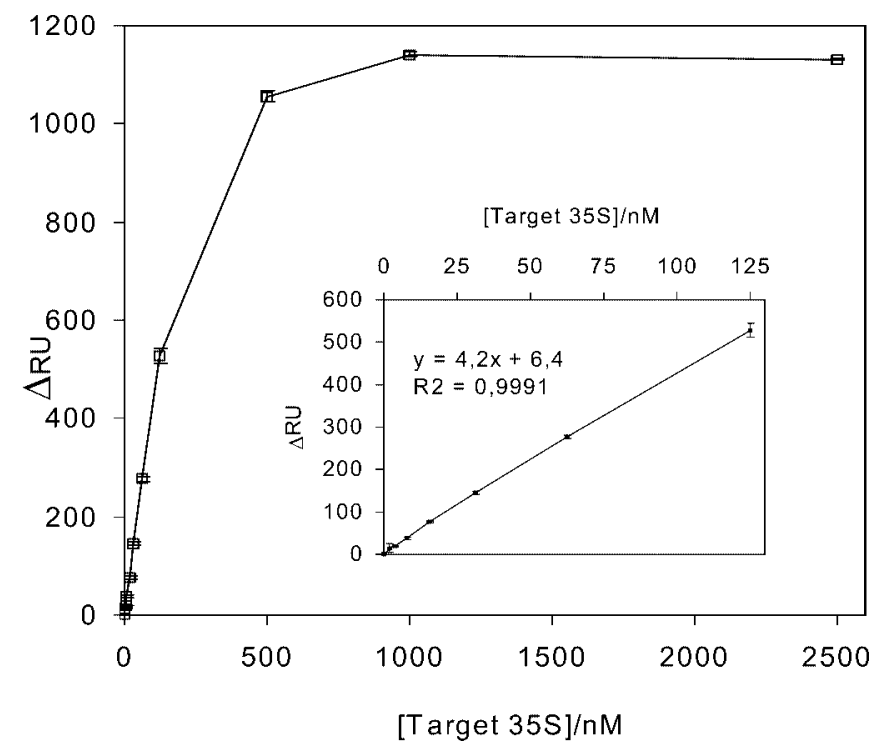

Fig. 8. SPR sensor: Calibration curve obtained with the 25-mer complementary oligonucleotides for P35Sprobe. In the right the zoom of the linear region is reported. The hybridisation reaction is allowed to proceed for 10'. All experiments were conducted using $25 \mu \mathrm{l}$ of DNA sample. In all the experiments the single stranded probe was regenerated by 1 min treatment with $1 \mathrm{mM}$ $\mathrm{HCl}$ allowing a successive hybridisation reaction to be monitored.

complementary oligonucleotides for the P35S are first constructed for both sensors. Their profiles are reported in Figs 7 and 8 respectively for QCM and SPR based system.

The specificity is confirmed by the absence of signal in both system when tested with 25 -mer noncomplementary oligonucleotides $(1.00 \mu \mathrm{M})$.

\subsubsection{Real sample testing}

PCR amplified samples from different sources of transgenic material from plasmidic, Certified reference material from flour, fresh plant and animal feeding can be also applied with both devices.

With PCR amplified sample (dsDNA) a denaturing step is necessary to obtain single stranded DNA (ssDNA) for the hybridisation reaction with the immobilised probe. The denaturing procedure can be performed using different approaches, i.e., thermal, thermal and chemical (using acidic and alkaline conditions), enzymatic (Enzymatic digestion of dsDNA amplified sequences using lambda $(\lambda)$ exonuclease, an enzyme that preferentially degrades one strand of duplex DNA), etc.

\subsubsection{Denaturing of DNA samples}

The most common denaturing method is the thermal one. The DNA samples are incubated at $95^{\circ} \mathrm{C}$ for 5 minutes, then transferred in ice for a further 1 minute. This method when applied to SPR and QCM sensing give very different results. In Mariotti et al. [5] it has been demonstrated that with the BiacoreX instrumentation the thermal treatment do not allow to obtain significant ssDNA reaching the sensor surface able to react with the probe. The reason of this behaviour, despite the high sensitivity encountered with synthetic oligonucleotides, relies on the fact that there is a spatial separation between the injection site where the sample is introduced and the sensor chip surface. So the flow carrying the sample takes a few seconds to reach the chip and during this time renaturation of the PCR complementary sequence, initially separated by the heat, reassociate. This problem can be overcome by employing a different denaturating method employing magnetic beads. With this new method the PCR amplicons are 


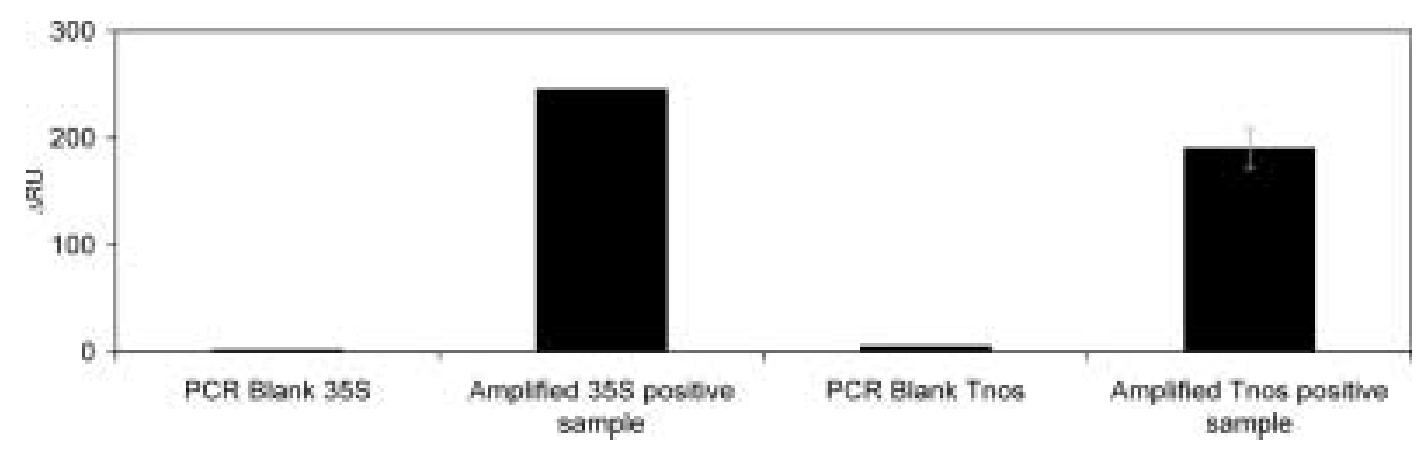

Fig. 9. SPR sensor: Results obtained with CRM from transgenic flour. The PCR amplified DNA was pre-treatment with magnetic particles coated with streptavidine to obtain the single sDNA carrying the target sequence. See text.

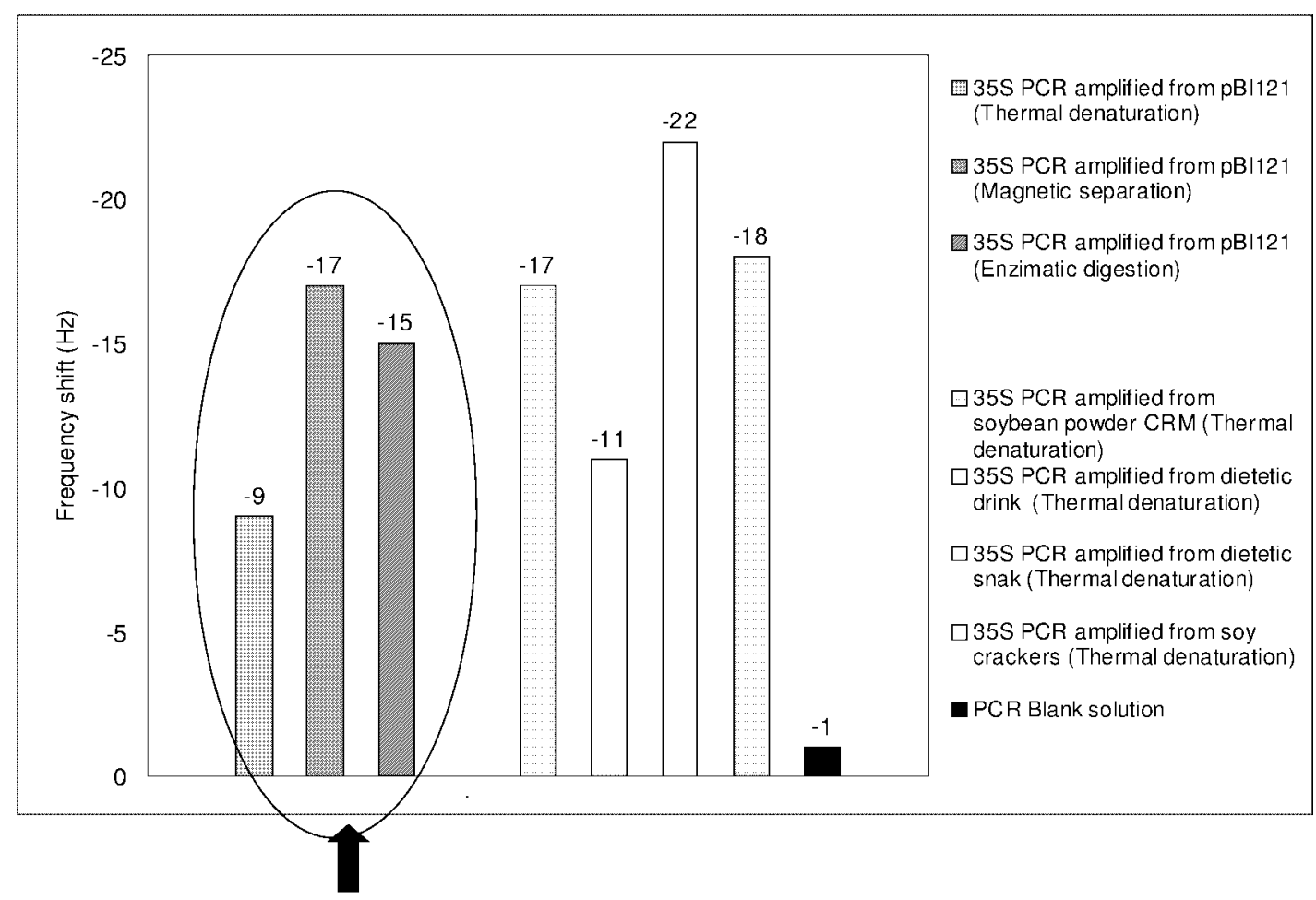

pBI121 treated with 3 different denaturating methods

Fig. 10. QCM sensor: Results obtained with different PCR amplified samples: plasmidic DNA from pBI121 (containing P35S) treated with three different denaturation methods (thermal, enzymatic and with magnetic particles), and transgenic CRM, dietetic snacks and light drinks (also containing P35S).

physical separated and no reannealing occurs after the injection of the sample. With this new method significant SPR signal can be recorded. In Fig. 9 is reported the resonance signal obtained with SPR instrument when P35 probe is immobilized on the surface and amplified P35S DNA treaded with the beads is added. The main disadvantages of this new method is the tedious procedure that increase the analysis time $(1 \mathrm{~h})$. Other denaturing methods are currently under study. 
The thermal method is, on the contrary, adequate for QCM DNA sensing. This method allows the detection of PCR amplified product in samples of very different origins (bacteria, human lymphocytes) as previously reported by Tombelli $[18,19]$ and also now with GMOs.

The main reason is that the measurement set-up is based on batch mode and when the sample is injected it reached immediately the probe allowing the hybridisation to occur. In this case, the analysis time is shortened and less tedious, if compared to the SPR system. In Fig. 10 are shown QCM signal obtained using three different denaturing methods, in particular, thermal, enzymatic and by magnetic beads, using PCR amplified for P35S (from plasmidic DNA). Signals obtained from different transgenic sample from different food origins, i.e., dietetic snacks and drinks, certified reference material, all treated only with thermal denaturing, are also reported.

\section{Conclusions}

Biosensors can be used for real-time hybridisation reaction monitoring, between an immobilised probe and target sequences in solution. Surface plasmon resonance (SPR) and piezoelectric (QCM) based sensors represent very interesting analytical devices for DNA detection. Many applications have been reported and here their use for genetically modified organism detection has been discussed. Both SPR and QCM can detected target sequence with high reproducibility of target sequences of P35S, the marker used for GMOs' detection, in adopted official methods. The specificity of these systems rely on the surfaces' immobilised probe sequences; changing probes, different target analytes (sequences) can be then detected.

These systems are very attractive for DNA sensing since their versatility is associated, often, with portability and with absence of any labelling. In addition many analysis can be performed on the same sensor surface, with the possibility to reuse the QCM and SPR devices, respectively, up to 30 times and more than hundred of times.

\section{References}

[1] A. Erdem, K. Kerman, B. Meric, U.S. Akarca and M. Ozsoz, DNA electrochemical biosensor for the detection of short DNA sequences related to the hepatitis B virus, Electroanalysis 11 (1999), 586-588.

[2] S. Löfas and B. Johnsson, A novel food hydrogel matrix on gold surfaces in surface plasmon resonance sensor for fast and efficient covalent immobilisation of ligands, J. Chem. Soc., Chemical Comm. 21 (1990), 1526.

[3] I. Mannelli, M. Minunni, S. Tombelli and M. Mascini, Quartz crystal microbalance (QCM) affinity biosensor for genetically modified organisms (GMOs) detection, Biosensor and Bioelectronics 18(2,3) (2003), 129-140.

[4] M.-P. Marco and D. Barcelò, Environmental applications of analytical biosensors, Measure Sciences Technology 7 (1996), $1547-1562$.

[5] E. Mariotti, M. Minunni and M. Mascini, Surface plasmon resonance biosensor for genetically modified organisms detection, Analytica Chimica Acta 453 (2002), 165-172.

[6] G. Marrazza, I. Chianella and M. Mascini, Disposable DNA electrochemical sensor for hybridisation detection, Biosensors and Bioelectronics 14 (1999), 43-51.

[7] M. Mascini, M. Minunni, G.G. Guilbault and R. Carter, Immunosensor based on piezoeelctric crystal devices in: Protocols in Biosensor Research, Humana Press, USA in the series Methods in Molecular Biotechnology (1998), 55-76.

[8] S.R. Mikkelsen, Electrochemical biosensors for DNA sequence detection, Electroanalysis 8 (1996), 15-19.

[9] M. Minunni and M. Mascini, Detection of pesticide in drinking water using real-time Biospecific Interaction Analysis (BIA), Analytical Letters 26 (1993) 1441-1460.

[10] M. Minunni, Simultaneous determination of $\beta 2$-microglobulin and Ig E using real-time BIA, Analytical Letters 28 (1995), 933-944.

[11] M. Minunni, M. Mascini, G.G. Guilbault and B. Hock, The quartz crystal microbalance as biosensor. A Status Report on its Future, Analytical Letters 28 (1995), 749-764. 
[12] M. Minunni, M. Mascini, R. Carter and G.G. Guilbault, A QCM dispalcement assay for Listeria monocytogenes, Anal. Chim. Acta 325 (1996), 169-174.

[13] M. Minunni, S. Tombelli, S. Pratesi, M. Mascini, P. Piatti, P. Bogani, M. Buiatti and M. Mascini, A piezoelectric affinity biosensor for Genetically Modified Organisms (GMOs) detection, Analytical Letters 34 (2001), 6.

[14] M. Minunni, S. Tombelli, E. Mariotti, M. Mascini and M. Mascini, Biosensors as new analytical tool for detection of Genetically Modified Organisms (GMOs), Fresenius' Journal of Analytical Chemistry 369 (2001), 589-593.

[15] S. Sawata, E. Kai, K. Ikebukuro, T. Lida, T. Honda and I. Karube, Application of peptide nucleic acid to the direct detection of deoxyribonucleic acid amplified by polymerase chain reaction, Biosensors and Bioelectronics 14 (1999), 397-404.

[16] S. Storri, T. Santoni, M. Minunni and M. Mascini, Surface modification methods for the development of piezoimmunosensors, Biosensor and Bioelectronic 13 (1998), 1795-1808.

[17] S. Tombelli and M. Mascini, Piezoelectric quartz crystal biosensor: Recent immobilisation schemes, Analytical Letters 33 (2000), 2129-2151.

[18] S. Tombelli, M. Mascini, C. Sacco and A.P.F. Turner, A DNA piezoelectric biosensor assay coupled with a polymerase chain reaction for bacterial toxicity determination in environmental samples, Analytica Chimica Acta 418 (2000), 1-9.

[19] S. Tombelli, M. Mascini, L. Braccini, M. Anichini and A.P.F. Turner, Coupling of a DNA piezoelectric biosensor and polymerase chain reaction to detect apolipoprotein E polymorphisms, Biosensors and Bioelectronics 15 (2000), 363-370.

[20] T. Vo-Dinh and B. Cullum, Biosensors and biochips: advances in biological and medical diagnostics, Fresenius Journal of Analytical Chemistry 366 (2000), 540-551.

[21] J. Wang, G. Rivas, X. Cai, E. Palecek, P. Nielsen, H. Shiraishi, N. Dontha, D. Luo, C. Parrado, M. Chicharro, P. Farias and F.S. Valera, DNA electrochemical biosensors for environmental monitoring: a review, Analytica Chimica Acta 347 (1997), 1-8

[22] J. Wang, G. Rivas and X.H. Cai, Screen-printed electrochemical hybridisation biosensor for the detection of DNAsequences from the Escherichia coli pathogen, Electroanalysis 9 (1997), 395-398.

[23] J. Wang, G. Rivas, J.R. Fernandes, J.L.L. Paz, M. Jiang and R. Waymine, Indicator-free electrochemical DNA hybridisation biosensor, Analytica Chimica Acta 375 (1998), 197-203. 


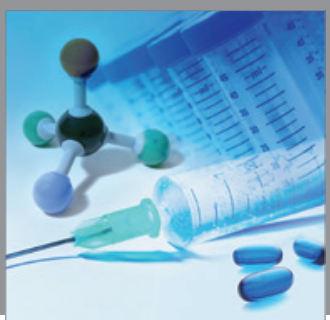

International Journal of

Medicinal Chemistry

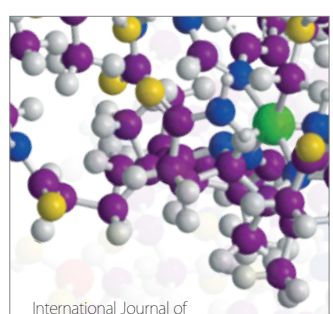

Carbohydrate Chemistry

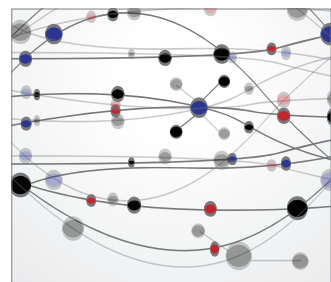

The Scientific World Journal
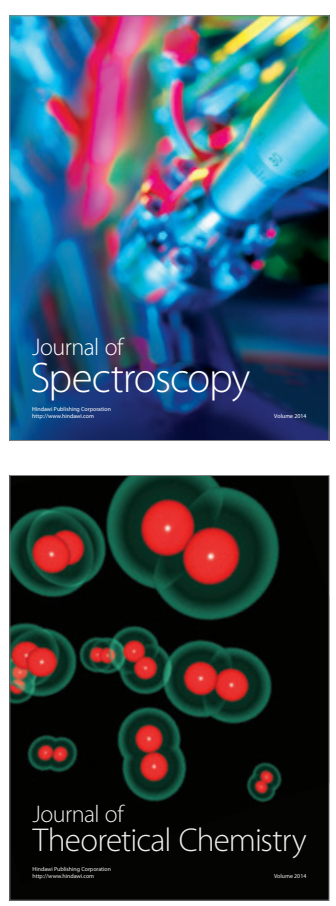
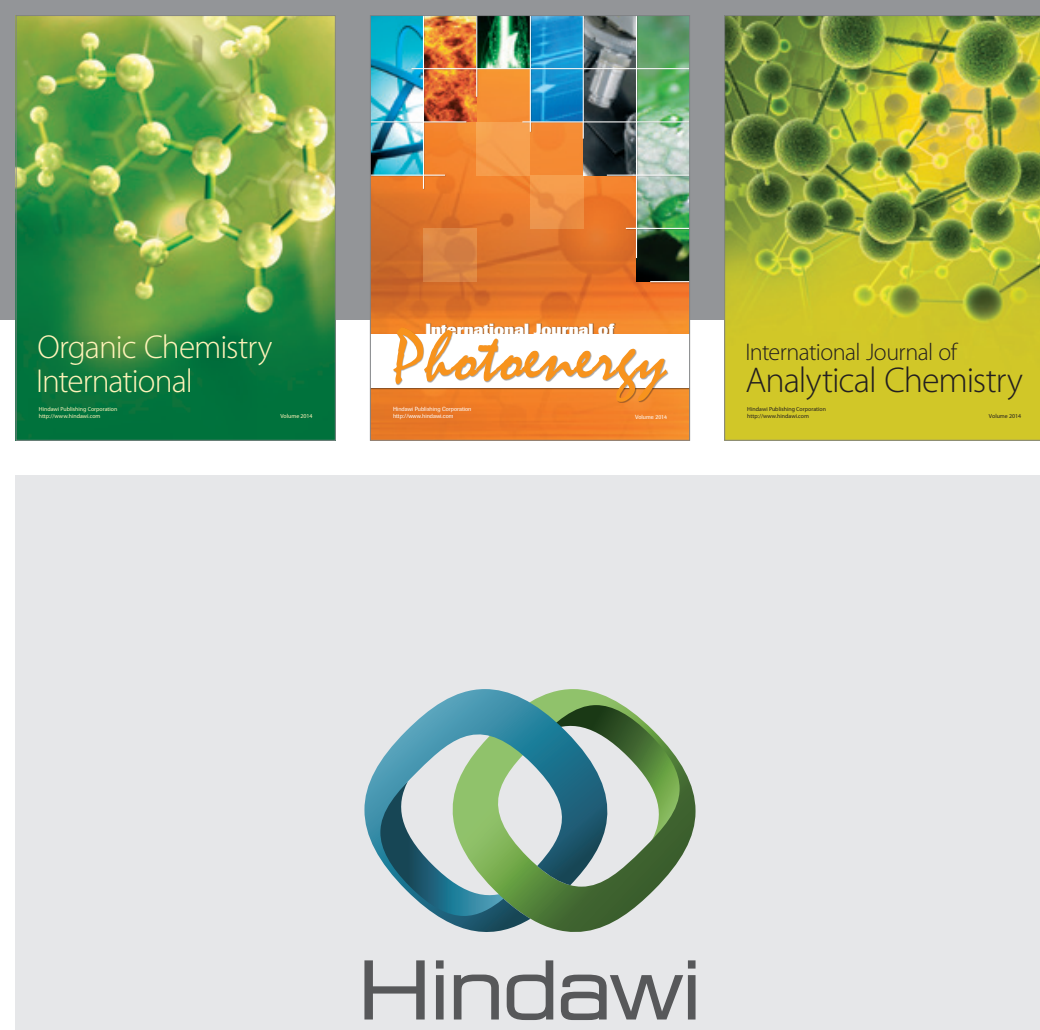

Submit your manuscripts at

http://www.hindawi.com
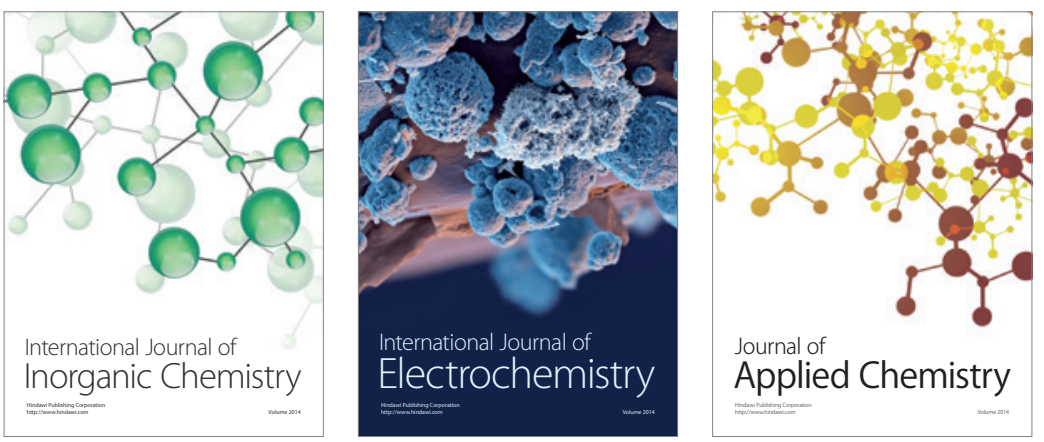

Journal of

Applied Chemistry
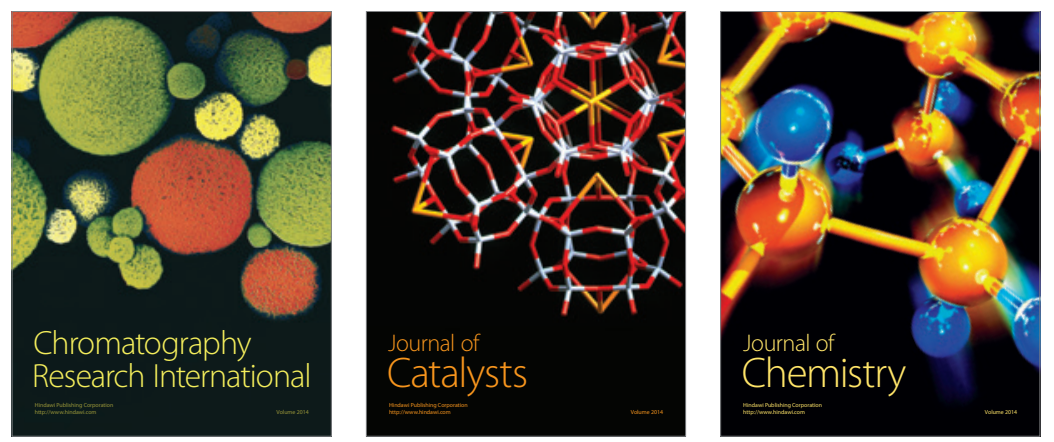
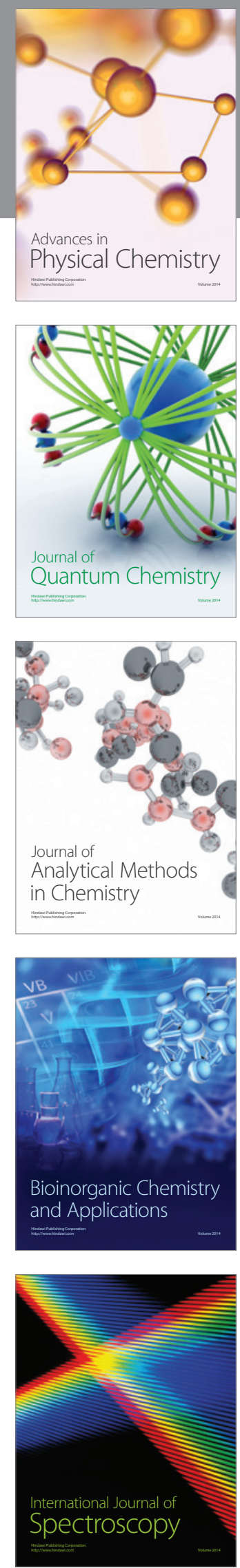